\title{
Chocs et survie dans l'éducation : le cas du Sénégal
}

\author{
Malick SANE ${ }^{a^{*}}$ \\ ${ }^{a}$ UCAD, Dakar, Sénégal \\ *malick.sane@ucad.edu.sn
}

\section{Résumé}

Les causes de l'échec scolaire dans les pays en développement ne sont pas uniquement liées au dysfonctionnement du système éducatif mais pourraient être associées à la qualité de vie environnementale et familiale dans laquelle évolue l'enfant. Cette étude est basée sur les modèles économétriques de durée. L'échantillon est composé d'enfants âgés entre 5 et 15 ans vivant dans des ménages ayant subi des chocs. Les résultats basés sur une analyse non paramétrique et ceux portant sur l'analyse paramétrique convergent vers les mêmes conclusions. Il ressort en effet de l'étude que, conditionnellement aux autres caractéristiques socio-économiques, les chocs négatifs diminuent les chances de survie à l'échec scolaire des enfants tandis que ceux positifs les augmentent. On note aussi que les autres caractéristiques socio-économiques (niveau de vie, niveau d'instruction, genre, localisation) impactent sur l'échec scolaire mais de manière différente. C'est pourquoi l'élaboration de mécanismes de réduction de la vulnérabilité des ménages est souhaitable. Cela peut passer par la mise en place de filets sociaux de sécurité par l'État ou par la promotion de systèmes de micro-assurance par les institutions de microfinance déjà présentes dans de nombreux pays.

Mots clefs : Chocs, Survie dans l'éducation, Echec scolaire.

\section{Shocks and survival in education: the case of Senegal}

\begin{abstract}
The causes of school failure in developing countries are not only related to the dysfunction of the education system but could be associated with the
\end{abstract}


environmental and family quality of life in which the child evolves. This study is based on econometric models of duration. The sample is made up of children aged 5 to 15 living in households that have experienced shocks. The results based on a nonparametric analysis and those on the parametric analysis converge to the same conclusions. The study shows that, conditional on other socio-economic characteristics, negative shocks reduce children's chances of surviving school failure while positive ones increase them. We also note that the other socioeconomic characteristics (standard of living, level of education, gender, location) have an impact on school failure, but in a different way. This is why the development of mechanisms to reduce the vulnerability of households is desirable. This may involve the establishment of social safety nets by the State or the promotion of microinsurance systems by microfinance institutions already present in many countries.

Keywords: Shocks, Survival in education, School failure.

\section{Introduction}

Dans un contexte de détérioration du niveau de vie et d'approfondissement des inégalités sociales, les populations des pays en développement, en général, celles du Sénégal, en particulier, affrontent périodiquement des chocs économiques. Parfois, il s'agit de chocs favorables comme la hausse de certains prix agricoles ou la réalisation de bonnes récoltes qui peuvent améliorer les conditions de vie des ménages, avec l'augmentation du revenu des parents et la valeur de leurs actifs. Ces chocs peuvent être des stimulateurs de dépenses notamment celles consacrées à l'éducation, afin d'optimiser les possibilités de réussite des enfants. Plus souvent, ces chocs sont néfastes comme dans le cas de sécheresse, d'inflation galopante, d'invasion acridienne, de chômage des parents de l'élève, de cas de maladie grave, de divorce de parents ou encore de décès d'un parent, etc. (Rodgers et Standing, 1981 ; Bonnet, 1993 ; Grootaert 1998 ; Basu et Van, 1998 ; Lachaud, 2007). Ce sont là des difficultés conjoncturelles qui peuvent influer sur le parcours de l'élève. 
Face à ces situations, les ménages éprouvent en général des difficultés à honorer un certain nombre de charges fixes, particulièrement celles liées aux dépenses scolaires. Tel est, par exemple le cas lorsqu'un ménage choisit le non enrôlement de ses enfants à l'école, la déscolarisation, l'arrêt temporaire des études ou le changement d'établissement, ce qui peut aboutir, par moment, à un échec scolaire. (Jacoby et Skoufias 1997 ; Bouaré et al., 2010). Les besoins de main-d'œuvre dans les économies basées sur l'agriculture, l'« informalisation » de ces économies et d'une manière générale la pauvreté constituent des facteurs économiques importants qui contribuent à la mise au travail des enfants en Afrique subsaharienne (Diallo, 2001; Dumas et Lambert, 2006; Kobiané et Marcoux, 2007).Plusieurs auteurs s'accordent pour dire que les enfants sont employés à cause de la pauvreté des parents géniteurs. Ils s'appuient sur l'idée selon laquelle les enfants ne peuvent pas fréquenter les écoles lorsque les parents sont pauvres (Rodgers et Standing, 1981 ; Bonnet, 1993 ; Grootaert 1998 ; Basu et Van, 1998 ; Lachaud, 2007). De la pauvreté découle alors la difficulté pour les défavorisés à investir dans le capital humain de leurs enfants, difficulté surtout financière. En fait, le pauvre doit faire face aux contraintes d'accès aux sources de financement de l'investissement dans le capital humain. En conséquence, la valeur du revenu ou du travail non payé, auquel il faut renoncer, explique en grande partie la faiblesse de la demande d'éducation des ménages pauvres au profit de la mise au travail des enfants. Beaucoup de travaux ont mis en évidence l'impact des chocs sociaux dans les performances scolaires des enfants. Une première approche consiste à penser que, dans un contexte où les marchés financiers sont efficients, le travail des enfants peut être utilisé pour lisser les chocs économiques. Dans ce cas, un choc négatif augmenterait la participation des enfants au travail (et éventuellement diminuerait leur participation à l'école), tandis qu'un choc positif pourrait retarder leur mise au travail. 
L’influence des caractéristiques familiales est également d'une grande importance. Les résultats de recherches indiquent que les enfants qui proviennent de familles désunies ou reconstituées, à faible revenu ou en dépendance économique, où il y a plusieurs enfants, et dont les parents sont peu scolarisés sont plus prédisposés à abandonner l'école (Bachman et al., 1971 ; Carins et al., 1989 ; Ekstrom et al., 1986 ; Elliott et Voss, 1974 ; Howell et Frese, 1982 ; Janosz et al., 1997).

C'est l'approche que retiennent Jacoby et Skoufias (1997). En effet, ils montrent sur données indiennes que les enfants de certains villages accumulent moins de capital humain du fait de chocs idiosyncrasiques ou de chocs macro-économiques, contre lesquels les individus ne peuvent s'assurer. Cependant, ils montrent aussi que le défaut de capital humain dû aux chocs, et qui aurait été évité si les individus pouvaient s'assurer, est très limité (de l'ordre de $2 \%$ sur 3 ans et demi).

L'approche de Guarcello, Mealli, et Rosati (2003) consiste à estimer directement l'impact de chocs, du rationnement du crédit et de l'assurance sur les choix d'allocation du temps des enfants. Les auteurs concluent que le rationnement du crédit pousse les enfants vers l'inactivité, tandis que les chocs les poussent vers le travail, sans nécessairement les faire sortir de l'école1. Une autre approche consiste à dire que le ménage peut être amené à faire travailler ses enfants car il y est contraint du point de vue productif alors même qu'il souhaiterait éviter de les faire travailler. Dans ce cas, un choc négatif sur la détention d'actifs productifs peut entraîner une baisse du niveau de travail enfantin. Bouaré et al. (2010) sur la base de l'Enquête légère intégrée auprès des ménages (ELIM) mènent une réflexion sur le même thème en limitant leur analyse au niveau de l'environnement familial.

\footnotetext{
${ }^{1}$ Christelle Dumas (2005), Offre de travail des enfants et demande d'éducation dans les pays d'Afrique de l'Ouest, Thèse de doctorat nouveau régime, Ecole des hautes études en sciences sociales, 
La littérature empirique sur ce thème est également bien fournie. Pour l'origine familiale de l'échec, le fait de perdre ses deux parents ou l'un(e) des deux semble avoir un effet désastreux sur la réussite scolaire des enfants. Dans une étude menée en 1995 en Ouganda, Manyire (1997) trouve que : « la majorité des filles ayant abandonné leurs études avaient leurs deux parents en vie, tandis que les garçons ayant abandonné avaient plus fréquemment leurs parents décédés. Ce qui signifie que la mort du père est plus susceptible de précipiter la fin prématurée de la scolarisation des garçons que celle des filles. Alors que la longévité du père n'empêche pas nécessairement les filles d'abandonner les études2».Flore Gubert, Anne-Sophie Rob/Ward et Jean-Noël Senne (2010) ont mené une analyse des liens entre décès et décisions de scolarisation en milieu rural malgache. Plus précisément, l'idée de ce travail est d'examiner si, dans un contexte où l'accès aux ressources est limité et où les mécanismes d'assurance formels et les marchés sont défaillants, les ménages soumis à des chocs inattendus affectant leurs ressources disponibles, comme le décès d'un de leurs membres, ont recours à des stratégies particulières de gestion des risques incluant l'arrêt de la scolarisation des enfants et l'augmentation du travail enfantin.

Abdoulaye Diagne, Ismaël Kafando, Moussa H. Ounteni, (2006)3, ont cherché à appréhender les facteurs qui influencent les abandons dans l'éducation primaire au Sénégal en estimant des modèles hiérarchiques multinomiaux afin d'identifier les facteurs d'abandons précoces.

\footnotetext{
${ }^{2}$ Flore Gubert, Anne-Sophie Rob/Ward et Jean-Noël Senne (2010)

${ }^{3}$ Abdoulaye Diagne, Ismaël Kafando, Moussa H. Ounteni, (2006), Pourquoi les enfants quittent-ils l'école ? Un modèle hiérarchique multinomial des abandons dans l'éducation primaire au Sénégal, CREA, Université de Dakar 
Toujours, au plan de l'impact de l'environnement scolaire sur le comportement individuel à l'école, les résultats de Rumberger (1995) militent en faveur d'effets modérateurs plus importants, confirmant ainsi les hypothèses de Moos (1979) sur l'influence de l'environnement scolaire.

Laurine Martinoty, (2013)4, se concentre sur les mécanismes intrafamiliaux d'offre de travail impliquant le conjoint comme travailleur additionnel en cas de choc économique touchant le pourvoyeur principal de revenu du travail dans le ménage. Appartenant à cette dernière catégorie, le «travailleur additionnel », défini par Woytinsky (1940) comme « la personne entrant sur le marché du travail suite à la perte d'emploi du principal pourvoyeur de revenu de sa famille, et qui n'aurait pas cherché du travail dans une autre situation », a suscité un vaste corpus de recherches empiriques aboutissant à des conclusions contradictoires ((Mincer [1962), Heckman et MaCurdy (1980, 1982), Maloney (1987, 1991), Lundberg (1985)).

L'éducation des enfants dans les pays en développement est une préoccupation majeure des gouvernements. Dans un contexte de détérioration du niveau de vie et d'approfondissement des inégalités sociales, les populations de ces pays affrontent périodiquement de brusques chocs économiques.

C'est notamment le cas du Sénégal où la situation est marquée par la faiblesse du Taux brut de scolarisation primaire (TBSP) qui s'élève à $84 \%$ dans le cycle primaire en 2015 selon une enquête de l'Agence nationale de la statistique et de la démographie (ANSD) contre $82,5 \%$ en $2005^{5}$. De surcroît, le redoublement et l'abandon scolaire qui

\footnotetext{
${ }^{4}$ Laurine Martinoty, 2013, Stratégie familiale de gestion des chocs : I'offre de travail des épouses en réponse aux fermetures d'entreprise en Argentine, GATE Groupe d'Analyse et de Théorie Économique Lyon-St Étienne, Université de Lyon, France.

${ }^{5}$ Cette enquête de l'ANSD dénommée « A l'écoute du Sénégal 2014 » est une opération menée auprès de 1500 ménages ordinaires qui ont accès au réseau de téléphonie mobile sur l'étendue du territoire national dont la campagne de collecte de l'enquêe de référence s'est déroulée entre les mois de novembre et décembre 2014.
} 
touchent particulièrement les premières années du primaire ont tendance à persister et à s'amplifier. De ce point de vue, le souci de faire progresser une partie importante des enfants scolarisés vers les cycles de niveau supérieur se pose avec acuité. Des programmes spécifiques sont mis en place pour réduire notamment les redoublements et les abandons scolaires. Le problème de la survie dans l'éducation est central dans la mesure où un enfant qui n'a pas terminé les quatre premières années de l'enseignement primaire (qui forment sans doute le minimum requis pour acquérir de façon permanente la maîtrise de la lecture, de l'écriture et du calcul), a peu de chances de devenir un adulte alphabétisé. Par conséquent, les jeunes qui abandonnent leurs études prématurément risquent davantage de ne pas acquérir les connaissances et les compétences dont ils ont besoin pour pouvoir participer pleinement au marché du travail. Cette situation entraîne également une perte pour la formation de capital humain pour le pays et une perte du point de vue de l'efficacité dans l'utilisation des ressources publiques mobilisées pour la scolarité primaire, dans la mesure où les dépenses engagées pour les élèves qui sortent du système après seulement quelques années de scolarité ne produiront pas les résultats escomptés.

Tel est le cas d'un pays comme le Sénégal dont le choix dans ce travail se justifie par le fait que malgré les importants efforts budgétaires consentis avec $35 \%$ du budget national consacrés à l'éducation, soit 6\% du Produit intérieur brut (PIB) du pays, le système éducatif sénégalais est marqué par une faible capacité à faire progresser une proportion importante d'élèves, du début à la fin d'un cycle, en raison des abandons et redoublements qui sont encore élevés, en raison, notamment des chocs socioéconomiques subis par les ménages.

Pour agir efficacement contre l'échec scolaire résultant de chocs ayant affectés la demande d'éducation de certains ménages, il faut au préalable savoir quelle est la 
nature de ces chocs socioéconomiques. Toute politique définie dans l'offre éducative en vue d'améliorer les performances du système éducatif qui ne tiendrait pas compte des facteurs sous-jacents au phénomène serait d'une faible efficacité.

L'objectif de cette étude est ainsi d'appréhender les principaux déterminants de la survie dans l'éducation des enfants issus des ménages ayant subis des chocs socioéconomiques qui ont des conséquences sur les performances scolaires dans le système éducatif au Sénégal.

L'originalité de ce travail se situe à trois niveaux. D'abord, il exploite les informations fournies par une base de données issue d'une enquête nationale sur les dépenses d'éducation des ménages au Sénégal qui permet de disposer d'une large gamme de variables susceptibles d'agir sur la décision d'abandonner les études dans un cycle avant terme. Ensuite, il tient compte de la transition de la scolarisation à l'échec scolaire. Le temps de scolarisation étant continu, cette transition est matérialisée par une variable continue qui mesure le temps entre le début de scolarisation et la sortie du système éducatif, qui est une rupture de cette scolarisation, étant donné que l'apprenant n'a pas encore dépassé l'âge scolaire. Enfin, l'application de modèles de durée ou de survie en présence de données censurées très performants lorsque l'on cherche à étudier le temps de sortie, l'échec, la survenance d'un événement chez un individu exposé à plusieurs facteurs comme des chocs.

Les autres développements sont organisés comme suit. La deuxième partie expose plus largement la méthodologie utilisée pour mieux cerner les déterminants des chocs sur la survie dans l'éducation des enfants, à savoir le modèle d'analyse et la méthode d'estimation. La troisième partie présente l'analyse empirique en considérant les données, les résultats et l'interprétation des résultats des estimations effectuées alors que la quatrième partie tire des conclusions de l'étude. 


\section{Méthodologie}

L'analyse de survie est une méthode statistique utilisée pour analyser la durée observée pour la réalisation d'un événement. Elle est retenue dans plusieurs domaines, notamment en ingénierie où l'on parle d'analyse de fiabilité, en démographie et en médecine, pour l'étude de durée de la vie humaine, de durée entre déclenchement d'une maladie et sa guérison. En économie ou en assurance, cette méthode permet de cerner la durée d'un épisode de chômage, la durée de vie d'une entreprise, la durée séparant deux sinistres, la durée avant la sortie d'un système, etc.

Parmi les pionniers de cette méthode d'analyse, on peut citer Weibull (1951), Kaplan et Meier (1958) et Cox (1972) dont certains modèles portent leurs noms. Weibull propose un modèle paramétrique pour calculer la fiabilité d'un système non réparable, Kaplan et Meier proposent dans le domaine médical un estimateur non paramétrique qui prend en compte les données censurées alors que Cox utilise un modèle semi paramétrique faisant intervenir des variables explicatives qui sont exogènes.

Dans cette étude, la transition de la scolarisation à l'échec scolaire est utilisée. Ainsi on pourra voir comment les facteurs socio-économiques agissent sur la sortie du système éducatif ou l'échec scolaire de l'enfant.

Il faut cependant noter que seule une partie des enfants ont connu l'échec et l'autre partie est toujours scolarisée au moment de la collecte des données de l'enquête. La trajectoire scolaire de chaque enfant de l'échantillon d'étude est connue seulement pour la période précédant l'enquête. On parle ainsi de données censurées à droite, l'information sur la scolarisation de l'enfant après cette date d'enquête du ménage n'est pas connue. Ceci justifie, en partie, l'application des modèles de durée ou de survie en présence de données censurées. 


\subsection{Le modèle d'analyse}

On considère que la durée de scolarisation de l'enfant est décrite par une variable aléatoire $T$ qui suit une distribution caractérisée par sa fonction de répartition $F(t, \theta)$. Cette fonction représente la probabilité de l'échec scolaire de l'enfant au temps $t$ ou avant cette date :

$$
F(t, \theta)=P(T<t)
$$

A cette fonction de répartition, on associe la densité $f(t, \theta)$ et la fonction de survie $S(t, \theta)$ qui représente la probabilité que la période de scolarisation de l'enfant dépasse la date ou bien que l'enfant survie à l'échec scolaire à la date t. Cette fonction s'écrit comme suit :

$$
S(t, \theta)=1-P(T<t)=P(T>t) .
$$

Une autre fonction utilisée est la fonction de risque instantané ou taux instantané ou la fonction de hasard $h(t, \theta)$ qui est définie comme étant la probabilité instantanée d'échec à la date t sachant que l'individu n'a pas encore connu l'échec.

$$
h(t, \theta)=\frac{f(t, \theta)}{S(t, \theta)}
$$

Selon la fonction de hasard, on a plusieurs sortes de fonction de survie. Ainsi on obtient le type de distribution suivi par la variable aléatoire $T$ : la loi exponentielle, la loi de Weibull, la loi log-normale et la loi log-logistique.

Pour savoir comment les caractéristiques socio-économiques $X$ influencent la probabilité instantanée d'échec scolaire d'un enfant et l'ampleur de cette influence, il s'avère nécessaire de faire dépendre cette fonction de hasard à ces variables socioéconomiques $X$. En utilisant le paramètre à estimer $b=(\theta, \beta)$, on introduit ces variables explicatives notées : 


$$
h(t)=h(X, t, b)
$$

Les modèles de durée peuvent être regroupés en deux classes : les modèles à risque proportionnel et les modèles à vie accélérée.

En supposant que les variables socio-économiques ont un impact multiplicatif sur la fonction de hasard, on se situe au niveau des modèles à risque proportionnel avec

$$
h(t)=h(X, t, b)=h_{0}(t / \theta) * \exp (X \beta)
$$

Avec $h_{0}(t / \theta)=h_{0}(t, X=0, \theta)$ la fonction hasard de base

Dans le cas où l'on suppose que l'impact multiplicatif est sur la variable de durée ou que l'impact additif est sur le logarithme de cette variable, on est en présence de modèles à hasard accéléré.

$$
h(t)=h(X, t, \theta)=h_{0}(t \exp (X \beta) / \theta) * \exp (X \beta) .
$$

Dans cette présente étude on suppose que l'impact sur la variable de durée est multiplicatif. On considère la variable aléatoire $T_{0}$ qui décrit la date ou le temps d'échec scolaire d'un enfant issu d'un échantillon homogène d'individus dont les caractéristiques socio-économiques correspondent à celles de variables ...lorsqu'elles prennent des valeurs nulles. La distribution de $T_{0}$ est aussi supposée être identique à celle de $h_{0}$. A l'instar de $T$, la variable $T_{0}$ peut avoir comme distribution la loi exponentielle, la loi de Weibull, la loi log-normale ou la loi log logistique.

Pour étudier comment les variables influencent le passage de la scolarisation à l'échec scolaire, on écrit le modèle comme suit :

$$
T=\exp (X b) * T_{0}
$$

En mettant cette dernière expression sous forme log-linéaire, on obtient :

$$
\log (T)=\mathrm{Xb}+\log \left(T_{0}\right)
$$


En posant $\log \left(T_{0}\right)=\varepsilon$, on obtient :

$$
\log (T)=\mathrm{Xb}+\varepsilon
$$

On estime ainsi le modèle en choisissant parmi les lois candidates pour $T_{0}$ celle la mieux adaptée.

\subsection{Echantillon et variables utilisées pour l'étude}

L'échantillon d'étude concerne dans ce travail les enfants âgés de 5 à 15 ans, intervalle d'âge supposé être celui correspondant à la scolarisation obligatoire. Ces enfants sont tous issus de ménages ayant subi un choc positif ou bien un choc négatif et sont au nombre 822 individus. Il est composé d'enfants ayant connu la scolarisation au moins pendant une année scolaire pour pouvoir parler d'échec scolaire ou pas.

Comme variable expliquée ou variable dépendante, on a la durée d'étude (duree_etude) qui mesure le temps, en nombre d'années, entre le début de scolarisation de l'enfant et sa sortie du système scolaire pour ceux qui ont connu l'échec scolaire, et pour les enfants n'ayant pas connu l'échec scolaire la dernière période correspond à l'année de l'enquête.

Parmi les variables explicatives nous avons :

Les chocs : les chocs négatifs subis par le ménage regroupent un ensemble de phénomènes négatifs affectant le ménage ou un membre du ménage : décès d'un membre de la famille, une blessure sérieuse ou une maladie empêchant un membre du ménage de faire correctement ses activités, une perte du travail régulier d'un membre du ménage, entre autres. Les chocs positifs peuvent se manifester sous la forme d'un nouveau travail régulier pour un membre du ménage, l'héritage, un gros don, un gain à la loterie, un versement important venant d'une entreprise (indemnités, dividendes, etc.), un mariage, des fiançailles, etc. La variable chocs est une variable dichotomique 
prenant la valeur 1 pour les individus vivant dans des ménages ayant subi au moins l'un des chocs positifs et elle prend 0 pour les individus dont le ménage a subi au moins l'un des chocs négatifs cités. Nous notons:

- La diminution des dépenses allouées à l'éducation de l'enfant (diminution_dep_scol) qui favorise l'échec scolaire de l'enfant. C'est une variable affectant directement la scolarisation de l'enfant.

- Les quintiles de niveau de vie (quintile1 à quintile 5) : ils classent les ménages dans cinq groupes différents à partir de leurs dépenses de consommation annuelle. Chaque classe regroupe $20 \%$ du total des ménages. Le premier quintile correspond aux $20 \%$ des ménages les plus pauvres, le deuxième aux $20 \%$ les moins pauvres que les premiers, ainsi de suite. Le dernier quintile regroupe les $20 \%$ des ménages les plus riches. On pourrait s'attendre à ce qu'un enfant qui se situe au niveau d'un quintile supérieur ait plus de chance de ne pas connaitre l'échec scolaire que celui se trouvant au niveau d'un quintile de niveau inférieur.

- Le sexe de l'enfant (sexe) : il est possible que l'échec scolaire affecte différemment l'enfant selon qu'il soit une fille ou un garçon ;

- L'âge : la sortie du système éducatif s'accélère avec l'âge ;

- Le sexe (femme) et l'âge du chef de ménage qui sont aussi des variables déterminant pour étudier l'échec scolaire ;

- Les variables géographiques Dakar, autres centres villes et le milieu rural. Le milieu géographique est une variable déterminante dans la scolarisation des enfants. En effet la scolarisation des enfants est plus importante en milieu urbain du fait de la proximité des infrastructures scolaires, entre autres ;

- Le travail effectué par l'enfant qui peut favoriser son échec du système scolaire ; 
- Le niveau d'éducation du chef de ménage : un niveau plus élevé est supposé s'associer avec une diminution de l'échec de l'enfant.

\section{Analyse empirique}

Les résultats présentés dans les parties qui suivent ont été obtenus en utilisant le logiciel STATA version 13.

\subsection{Analyse non paramétrique}

L'analyse non paramétrique est basée sur l'estimation non paramétrique de KaplanMeier de la fonction de survie qui est la distribution suivant le temps de la probabilité de ne pas constater l'échec scolaire d'un enfant avant une date t. La fonction de survie a une représentation en escalier et donc chaque plateau de la courbe représente la probabilité de ne pas connaitre l'échec scolaire durant la période de temps (en abscisse) délimitant le plateau. Il s'agit ici, entre autres, de faire une analyse globale puis une analyse comparative des différentes fonctions de survie selon les groupes.

\subsubsection{Fonction de survie globale}

Le graphique 1 donne une représentation de la fonction de survie de l'ensemble de l'échantillon de l'étude. On note une décroissance rapide de la fonction durant les sept années de scolarisation. Au-delà de cette période, on remarque un ralentissement de cette décroissance de la fonction qui est, presque, stable après 15 ans. Ainsi on peut dire, pour notre échantillon global, que la chance de ne pas connaitre l'échec scolaire pour un enfant diminue de façon considérable au fil des sept années de scolarisation qui correspondent, en référence au système éducatif national sénégalais, à l'école primaire et à la première année du niveau moyen. 
Figure 1: Fonction de survie globale

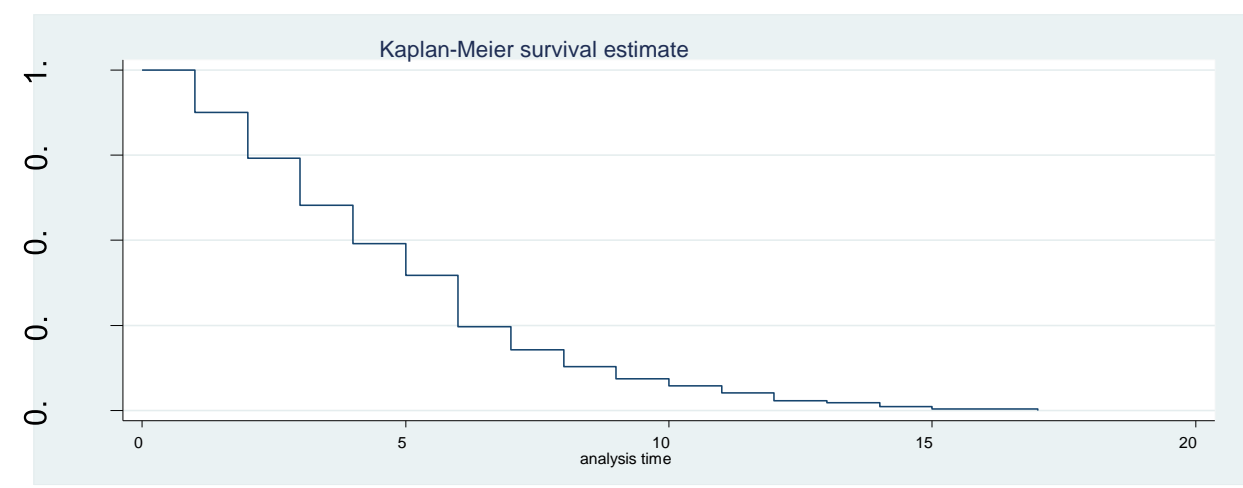

Source : calcul de l'auteur à partir de la base

\subsubsection{Analyse de la survie suivant les groupes}

On s'intéresse d'abord aux effets de différents chocs sur la fonction de survie. Le graphique 2 suivant donne le comportement de la fonction de survie lorsqu'on est en présence de deux groupes : ceux ayant subi des chocs négatifs et ceux qui ont vécu des chocs positifs.

Figure 2: Survie suivant les chocs

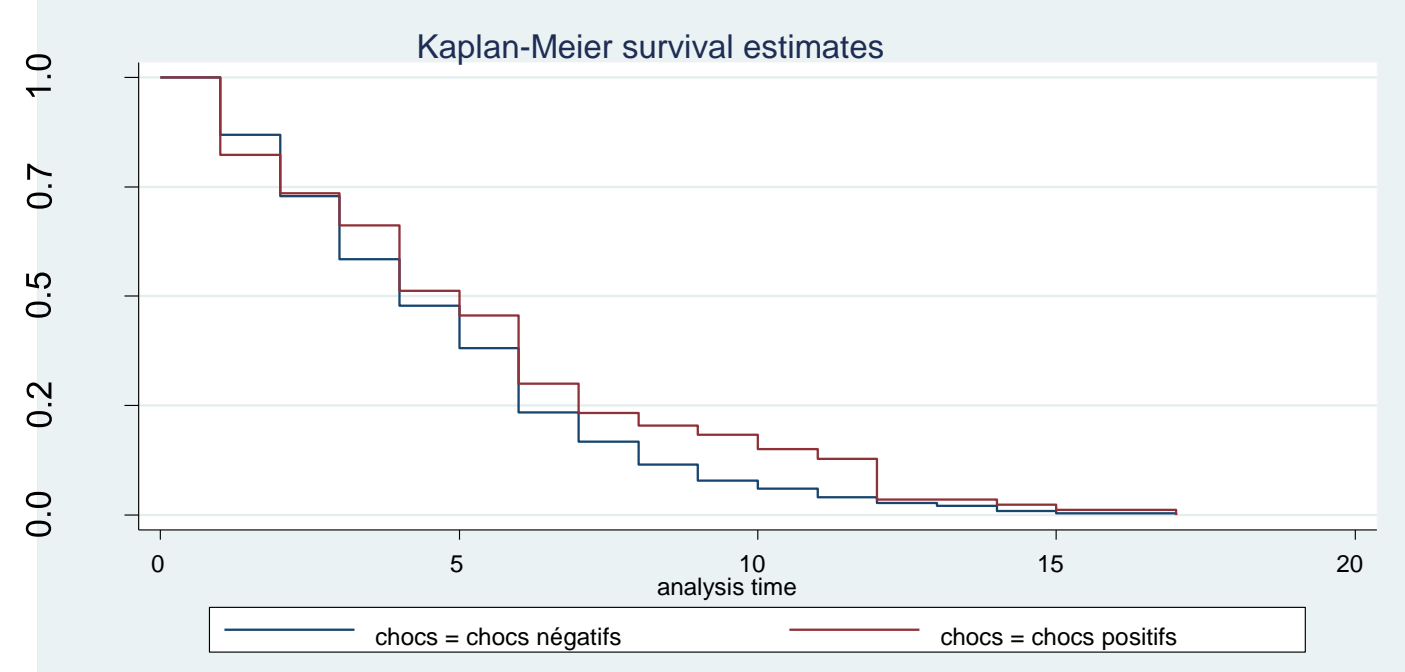

Source : calcul de l'auteur à partir de la base

Sur le graphique 2, à l'image de la fonction de survie globale, on remarque une décroissance assez forte puis régulière des fonctions de survie des deux groupes, ceux ayant subi des chocs négatifs (en bleu) et ceux ayant subi des chocs positifs (en rouge). 
Pour presque toute la période correspondant à la durée de scolarisation, la probabilité de survie à l'échec scolaire des enfants ayant connu des chocs négatifs dans leurs ménages reste inférieure. Ceci aurait comme explication le fait que les enfants ayant connu les chocs négatifs sont plus exposés à l'échec scolaire durant la durée de scolarisation. Ainsi ceux vivant dans des ménages ayant connu des chocs positifs ont plus de chance de ne pas connaitre l'échec comparativement à ceux qui ont connu des chocs négatifs.

\subsubsection{Les différents quintiles de niveau de vie}

Le graphique 3 nous donne l'allure de la fonction de survie pour chaque quintile de niveau de vie. On note que l'allure de la probabilité de survie à l'échec scolaire en fonction du nombre d'année est presque identique pour tous les quintiles. La probabilité de sortie du système scolaire avec le temps semble ne pas avoir un écart significatif entre les quatre premiers quintiles. Dans ce cas, il serait nécessaire de faire une analyse paramétrique conditionnellement aux variables explicatives pour bien conclure.

Toutefois il faut noter que la survie à l'échec scolaire du cinquième quintile reste supérieure à toutes les autres indépendamment des caractéristiques socio-économiques des enfants.

Figure 3: Survie suivant les différents quintiles de niveau de vie

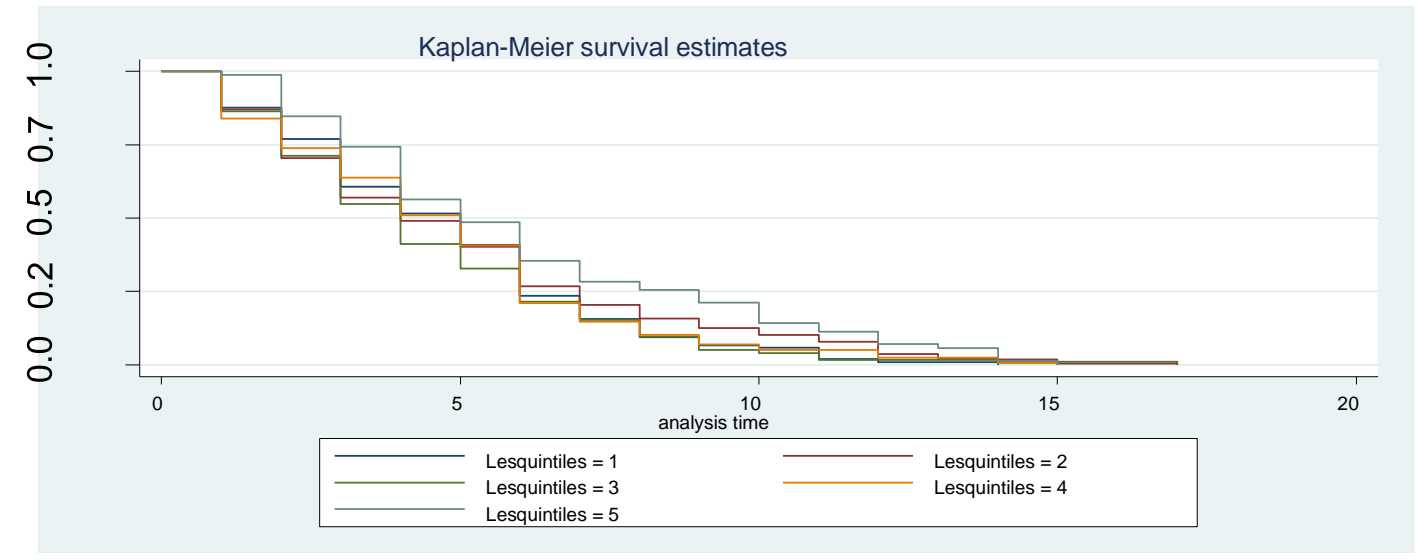

Source : calcul de l'auteur à partir de la base 


\subsubsection{Analyse suivant le genre}

La probabilité de survie que l'enfant soit une fille ou un garçon a une allure presque identique. Une analyse plus fine de la fonction de survie montre que l'échec scolaire chez la fille est plus probable pendant les dernières périodes de la durée de scolarisation. Au total, l'échec scolaire semble affecter de manière différente les garçons et les filles selon les différentes périodes du cycle scolaire comme le montre le graphique 4.

Figure 4: Analyse suivant le genre

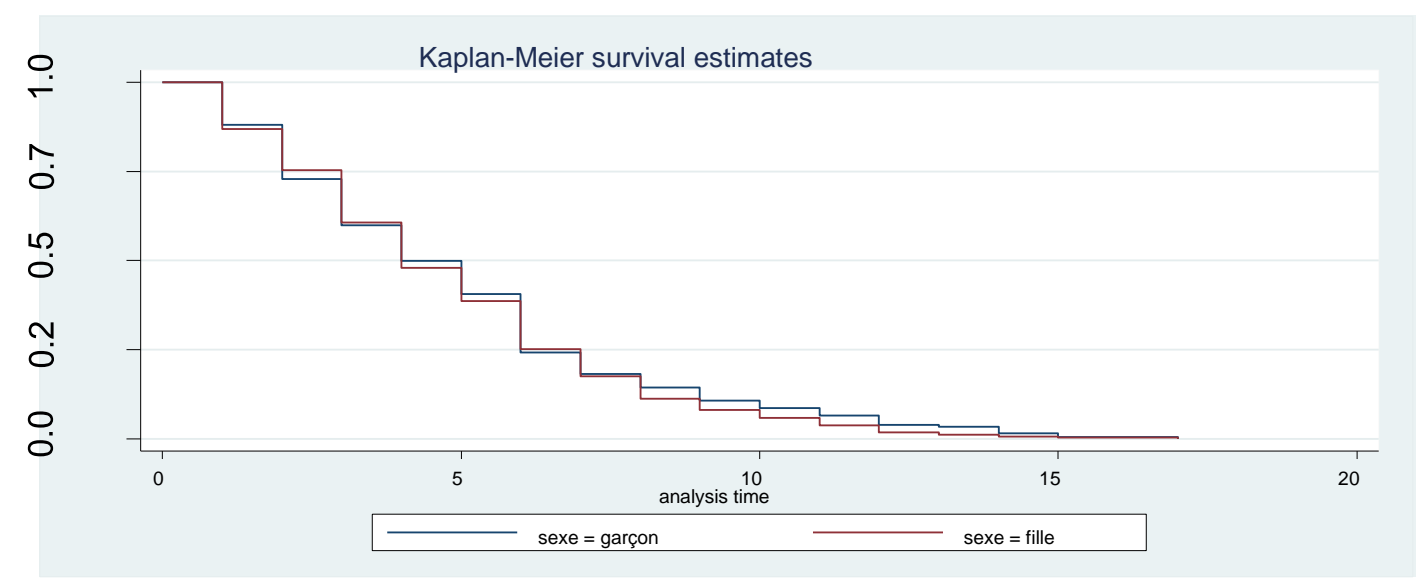

Source : calcul de l'auteur à partir de la base

\subsubsection{Analyse selon le chef de ménage}

Le graphique 5 fait une représentation des différentes fonctions de survie à l'échec scolaire selon qu'on est dans un ménage avec un chef de ménage femme ou un chef de ménage homme. 
Figure 5 : Source : Survie en fonction du genre du chef de ménage

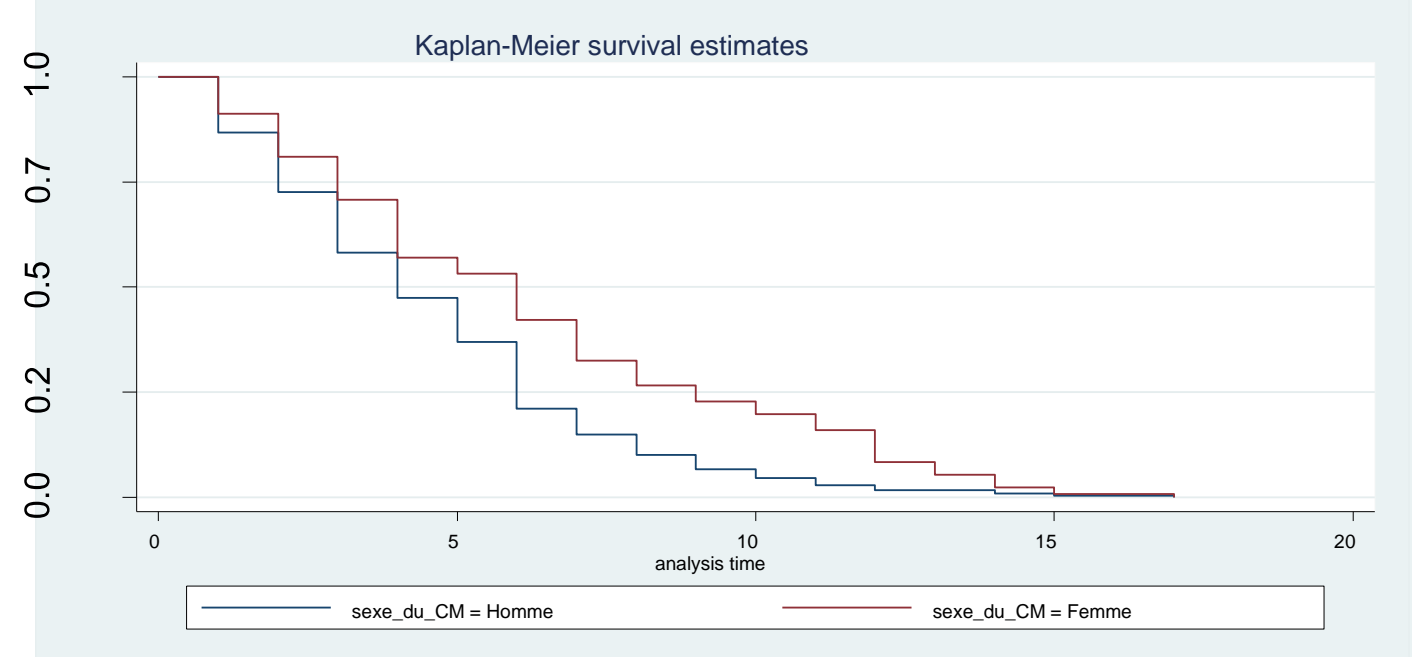

Source : calcul de l'auteur à partir de la base

On note à travers le graphique 5 que durant toute la période de la durée de scolarisation, la fonction de survie des enfants se trouvant dans un ménage avec chef de ménage femme est supérieure à celle des autres vivant avec un chef de ménage homme. Ainsi, les enfants vivant avec un chef de ménage homme sont les plus exposés à l'échec scolaire.

\subsection{Analyse paramétrique}

Dans cette partie, il est question d'analyser les différents résultats issus de l'estimation de notre modèle économétrique. Pour ce faire, il est nécessaire de choisir une loi de base pour estimer ce modèle. Un test basé sur la comparaison de l'AIC (Akaike information criterion) des différentes estimations du modèle est souvent utilisé pour retenir cette loi. De ce fait, le modèle qui sera retenu sera celui qui aura l'AIC le plus petit.

En se basant sur ce critère de sélection de la loi de base, on choisit la loi lognormale $(\mathrm{AIC}=1888,65)$ parmi les quatre proposées. Nous allons donc présenter les résultats issus de l'estimation économétrique du modèle avec, comme loi de base, la loi $\log$ normale. 
Dans le tableau ci-dessous, les valeurs des coefficients $b$ du modèle estimé ne sont pas présentées mais plutôt l'exponentiel $\exp (b)$ des valeurs pour chaque coefficient, qui correspond au taux de hasard ou risque instantané d'échec scolaire ou taux instantané d'échec scolaire. Sauf mention contraire, les seuls coefficients significatifs au seuil de $5 \%$ seront analysés. La dernière colonne permet de faire l'analyse en termes de pourcentage avec la modalité de référence des variables qualitatives. Les coefficients non significatifs sont statistiquement égaux aux coefficients des modalités de référence et donc l'écart est nul.

Tableau 1 : Résultats de l'estimation du modèle

\begin{tabular}{|c|c|c|c|c|}
\hline - t (durée) & Coef & z & $\mathrm{P}>|\mathrm{z}|$ & $\begin{array}{l}\text { Pourcentage } \\
\text { (coef- } \\
\text { coef_référence) }\end{array}$ \\
\hline Quintile_16(référence) & 1 & & & \\
\hline Quintile_2 & 0.1980583 & 2.69 & 0.007 & $-80 \%$ \\
\hline Quintile_3 & 0.1803009 & 2.37 & 0.018 & $-82 \%$ \\
\hline Quintile_4 & 0.1227476 & 1.54 & 0.124 & $0 \%$ \\
\hline Quintile_5 & 0.3687454 & 3.55 & 0.000 & $-63 \%$ \\
\hline $\begin{array}{l}\text { Chocs (Négatifs } \\
\text { référence) }\end{array}$ & 1 & & & \\
\hline
\end{tabular}

${ }^{6}$ Le premier quintile de niveau de vie constitue le quintile de référence dans l'analyse 
The Journal of Quality in Education (JoQiE) Vol.11, N¹7, May 2021

\begin{tabular}{|c|c|c|c|c|}
\hline Chocs (Positifs ici) & 0.1576384 & 2.01 & 0.044 & $-84 \%$ \\
\hline Sexe(garçon référence) & 1 & & & \\
\hline Sexe(Fille) & 0.0813042 & 1.57 & 0.116 & $0 \%$ \\
\hline âge & 0.0915502 & 16.83 & 0.000 & \\
\hline diminution_dep_scol & $4.82 \mathrm{e}-07$ & 1.23 & 0.219 & \\
\hline $\begin{array}{l}\text { sexe_du_CM(Homme } \\
\text { référence) }\end{array}$ & 1 & & & \\
\hline sexe_du_CM(Femme) & 0.3489286 & 4.43 & 0.000 & $-65 \%$ \\
\hline Rural(référence) & 1 & & & \\
\hline Dakar & 0.1815085 & 1.74 & 0.082 & $-82 \%$ \\
\hline Autre centres villes & 0.013378 & 0.22 & 0.828 & $0 \%$ \\
\hline Enfant travaille(référence) & 1 & & & \\
\hline Ne travaille pas(enfant) & -0.1047478 & -1.36 & 0.173 & $0 \%$ \\
\hline CM_aucun_niveau(référence) & 1 & & & \\
\hline CM_niveau_primaire & 0.0046786 & 0.03 & 0.973 & $0 \%$ \\
\hline CM_niveau_moyen_secondaire & 0.1918977 & 2.92 & 0.004 & $-81 \%$ \\
\hline CM_superieur_professionel & 0.2493016 & 2.80 & 0.005 & $-75 \%$ \\
\hline
\end{tabular}

Source : calcul de l'auteur à partir de la base

Pour les variables relatives aux quintiles de niveau de vie, on remarque que,

conditionnellement aux autres variables explicatives, le taux instantané d'échec scolaire est supérieur au niveau du premier quintile qui correspond aux $20 \%$ des ménages les plus pauvres. En fait, le taux instantané d'échec scolaire du premier quintile est de 
presque $80 \%$ supérieur à celui du deuxième quintile, $82 \%$ supérieur à celui correspondant au troisième quintile et $63 \%$ à celui du cinquième quintile. Globalement on note que les enfants vivant dans le premier quintile ont moins de chance de ne pas connaitre l'échec scolaire que ceux des autres quintiles. Ces résultats confirment ceux des travaux de Bachman et al., 1971 ; Carins et al., 1989 ; Ekstrom et al., 1986 ; Elliott et Voss, 1974 ; Howell et Frese, 1982 et de Janosz et al., 1997.

Quant aux différents types de chocs vécus par les ménages, on remarque que le taux instantané d'échec scolaire s'accentue considérablement avec les ménages ayant subis les chocs négatifs. On note que les ménages ayant subis ces chocs négatifs ont $84 \%$ plus de risque de connaitre de l'échec scolaire en un moment donné que les ménages ayant connu des chocs positifs. On peut donc dire que l'impact des chocs négatifs accélère la sortie du système éducatif plus que celui des chocs positifs.

Une augmentation de l'âge va dans le sens d'accroitre le risque instantané d'échec scolaire des enfants.

En ce qui concerne l'analyse suivant le chef de ménage, on remarque que les résultats obtenus confirment ceux présentés dans l'analyse non paramétrique. Les enfants vivant dans des ménages sous la tutelle d'un chef de ménage femme ont un taux instantané d'échec scolaire à 65\% inférieur à celui des enfants vivant dans des ménages dirigés par des hommes.

Au seuil de $10 \%$, les enfants se trouvant dans la région de Dakar font face à un risque instantané d'échec scolaire largement inférieur (moins $82 \%$ ) à celui des enfants issus du milieu rural. Cependant, on ne constate pas un écart significatif entre les taux instantanés d'échec scolaire des enfants vivant dans les autres centres villes et ceux issus du milieu rural. Le risque instantané d'échec scolaire est identique chez les enfants vivant dans des ménages avec des chefs de ménage n'ayant aucun niveau d'instruction 
et ceux vivant avec des chefs de ménage avec un niveau primaire. Cependant, le taux instantané d'échec scolaire des enfants qui se trouvent dans des ménages avec un chef de niveau moyen ou secondaire est inférieur de $81 \%$ à ceux vivant dans des ménages avec un chef sans aucun niveau d'instruction. Pour les enfants vivant dans des ménages avec un chef de ménage d'un niveau supérieur ou professionnel, ce taux est de $75 \%$. On peut conclure à partir de là qu'un niveau plus élevé d'instruction du chef de ménage va dans le sens de diminuer le taux instantané d'échec scolaire des enfants. Diagne, Kafando et Ounteni (2006) aboutissent aux mêmes conclusions.

\section{Conclusion}

Les statistiques recueillies à partir de l'Enquête nationale sur les dépenses d'éducation des ménages au Sénégal procurent un certain éclairage sur le rôle des chocs socioéconomiques dans les performances scolaires de l'enfant. Elles mettent en évidence l'ampleur et les différentes formes de chocs subis par les ménages et soulignent l'interférence des caractéristiques des ménages concernés dans la survie de l'enfant dans le système éducatif. Les données montrent comment les facteurs socioéconomiques agissent sur la sortie du système éducatif ou l'échec scolaire de l'enfant. Le recours à un modèle de durée ou de survie en présence de données censurées est très adapté lorsque l'on veut étudier le temps de sortie, l'échec, la survenance d'un événement chez un individu, etc., si ce dernier est exposé à plusieurs facteurs comme des chocs.

L'analyse non paramétrique révèle que l'enfant ayant connu un choc négatif est plus exposé à l'échec scolaire que celui vivant dans un ménage ayant connu un choc positif qui a plus de chance de ne pas connaitre l'échec que le premier. Quand on considère le niveau de vie, on observe que la survie à l'échec scolaire du cinquième quintile reste supérieure à tous les autres indépendamment des caractéristiques socio- 
économiques de l'enfant. De l'analyse fondé sur le genre, il ressort que l'échec scolaire chez la fille est plus probable pendant les dernières périodes de la durée de scolarisation. Cette analyse menée suivant le chef de ménage montre que l'enfant dont le chef de ménage est un homme a moins de chance de survie dans l'éducation. Ces premiers résultats sont confirmés par ceux issus de l'estimation de notre modèle économétrique. Ainsi, les ménages ayant connu un choc négatif ont $84 \%$ plus de risque de connaitre de l'échec scolaire en un moment donné que les ménages ayant connu des chocs positifs. Autrement dit, on peut affirmer que l'impact des chocs négatifs accélère plus la sortie du système éducatif que celui des chocs positifs. En se référant au niveau de vie des ménages, on remarque que le taux instantané d'échec scolaire est plus élevé au niveau du premier quintile qui correspond aux $20 \%$ des ménages les plus pauvres. Par contre l'analyse de genre montre que l'enfant vivant dans un ménage sous la tutelle d'un chef de ménage femme a un taux instantané d'échec scolaire -5\% inférieur à celui de l'enfant vivant dans un ménage dirigé par un homme. L'analyse à partir de la localisation laisse apparaître qu'au seuil de 10\%, l'enfant se trouvant dans la région de Dakar fait face à un risque instantané d'échec scolaire largement inférieur (moins 82\%) à celui de l'enfant issu du milieu rural alors que celle considérant le niveau d'instruction permet de dire que plus élevé est niveau d'instruction du chef de ménage plus faible est le taux instantané d'échec scolaire de l'enfant.

Ces résultats montrent l'importance de mettre en place des mécanismes permettant de réduire la vulnérabilité des ménages, particulièrement pour ceux résidant en milieu rural afin d'atténuer l'impact des chocs négatifs dans les ménages des pays en développement confrontés à la pauvreté et à une plus grande exposition par rapport aux chocs socioéconomiques qui ont des conséquences négatives dans la survie dans l'éducation de milliers d'enfants. Cela peut passer par la mise en place de filets sociaux 
de sécurité par l'État ou par la promotion de systèmes de micro-assurance par les institutions de microfinance déjà présentes dans de nombreux pays.

\section{Références bibliographiques}

Abdoulaye Diagne, Ismaël Kafando, Moussa H. Ounteni, (2006), Pourquoi les enfants quittent-ils l'école ? Un modèle hiérarchique multinomial des abandons dans l'éducation primaire au Sénégal, CREA, Université de Dakar.

Akresh, (2006), School enrolment impacts of non-traditional household structure, BREAD Working paper $\mathrm{n}^{\circ} 89$.

Ambapour S., Mbko Ibara S., (2012), Survie des enfants et pauvreté au Congo : application d'un modèle de durée, Bureau d'Application des Méthodes Statistiques et Informatiques, 31p.

Best Francine, (1997), L'échec scolaire, Que sais-je, PUF.

Boure Issa et al., (2010), Environnement économique et éducatif des ménages et échec scolaire des enfants au Mali, CEPS INSTEAD Working Paper, $\mathrm{N}^{\circ}$ 2010-02, Février 2010, 36 pages.

Christelle Dumas (2005), Offre de travail des enfants et demande d'éducation dans les pays d'Afrique de l'Ouest, Thèse de doctorat nouveau régime, Ecole des hautes études en sciences sociales.

Cox D. R, (1972), Regression models and life-tables, Journal of the Royal Statistical Society, Vol. 34, $\mathrm{N}^{\circ} 2$.

El Hioui, M., Soualem, A., A.O.T., Aboussaleh, Y., Rusinek, S., Dik, K., (2008),

Caractéristiques socio-démographiques et anthropométriques en relation avec la performance scolaire dans une école rurale de la ville de Kenitra (Maroc), Antropo, 17, 25-33.

Elsayed A. Elsherpieny, Sahar A. N. Ibrahim, Noha U. M. M. Radwan, (2013), Discriminating between Weibull and Log-logistic distributions, International Journal of Innovative Research in Science, Engineering and Technology (ISO 3297: 2007 Certified Organization) Vol. 2, Issue 8, August, 14 p.

Gimeno José B., (1984), L'échec scolaire dans l'enseignement primaire : moyens de le combattre, études et enquêtes d'éducation comparée, UNESCO.

Grifa H., (2007), Retard de scolarisation au Bangladesh : une analyse économétrique, Centre d'économie de la Sorbonne, 29 p. 
Guarcello, L., Mealli, F., Rosati, F., (2003), Household vulnerability and child labor: The effect of shocks, credit rationing and insurance.

Hunter S., Williamson J., (2000), Children on the brink 2000: Updates estimates and Recommendations for intervention, USAID/The Synergy Project.

Jacoby, H., Skoufias, E., (1997), Risk, financial markets and human capital in a developing country, Review of Economic Studies 64, 311-335.

Jean-Pierre Lachaud, (2007), La mesure de la croissance pro-pauvres au Burkina Faso : espace de l'utilité ou des capacités ? Revue d'Economie du Développement, Vol. 15.

José Blat Gimeno, (1984), L'échec scolaire dans l'enseignement primaire : moyens de le combattre, Etude comparée internationale.

Kaplan E. L. and Paul Meier, (1958), Nonparametric Estimation from Incomplete Observations, Journal of the American Statistical Association, Vol. 53, $\mathrm{N}^{\circ} 282$.

Morice E., (1996) Quelques modèles mathématiques de durée de vie, Revue de statistique appliquée, tome 14, $\mathrm{n}^{\mathrm{0}} 1, \mathrm{p} .45-126$.

Soualem, A, Aboussaleh, Y., Ahami, A.O.T, Sekat, N.Yakrib, S., Zouiten, F., Azzaoui, F-Z.,

Rusinek, S., (2005), Impact du statut socioéconomique sur le développement cognitif et comportemental chez l'enfant scolarisé au Maroc. Journal de Thérapie Comportementale et Cognitive.15, 55-60.

Waloddi Weibull, (1951), A statistical distribution function of wide applicability, Journal of Applied Méchanics, September. 